\title{
The Design and Implementation of Professional Quality Evaluation System for Oil Workers in Oil Gathering and Transferring Enterprise
}

\author{
Huan Cheng-lin ${ }^{1}$, Chen Jian-wei ${ }^{2, *}$ \\ ${ }^{1}$ College of computer science, Yangtze University, No.1 Nanhuan Road, Jingzhou, China \\ ${ }^{2}$ Yangtze University College of Technology \& Engineering, No.85 Xueyuan Road, Jingzhou, China \\ *Corresponding author: webhcl@sina.com \\ Received December 03, 2014; Revised December 19, 2014; Accepted December 29, 2014
}

\begin{abstract}
From the realistic problems in the staff skills training assessment and professional quality requirement, design and develop professional quality appraisal system for oil gathering and transferring enterprise by integrated application of network technology and virtual simulation technology. System is in the service of oil workers professional quality requirement and regular training appraisal, realize the two aspects of theoretical knowledge and operation skills examination, and satisfy the unified examination of enterprise organization, and satisfy the employee's independent test.
\end{abstract}

Keywords: professional quality evaluation, evaluation system, virtual simulation

Cite This Article: Huan Cheng-lin, and Chen Jian-wei, "The Design and Implementation of Professional Quality Evaluation System for Oil Workers in Oil Gathering and Transferring Enterprise." Journal of Computer Sciences and Applications, vol. 2, no. 3 (2014): 44-47. doi: 10.12691/jcsa-2-3-2.

\section{Introduction}

Post skills training assessment and Professional skill appraisal becomes an important work in Oil gathering and transferring enterprise because staff liquidity is strong, change jobs frequently. Traditional way of examination not only requires a lot of manpower, financial and material resources, and it is very difficult to implementation, but the assessment has little effect. Building a digital staff professional quality inspection system, it implements enterprise total online training, evaluation, changes the past isolated closed training model, overcomes many disadvantages of concentrated examination, and provide powerful support services for training effect evaluation and professional skill appraisal.

\section{The Present Situation of the Oil Workers Professional Quality Assessment Implementation}

\subsection{The Theory Examination is Digital, but the Lack of System Planning}

The current staff training evaluation is based on computer system, but is just on the way to realize the digitization. Computer is to replace artificial to complete the roll, paper test, trainer reviews, etc. There is no application of modern education technology philosophy system planning, and evaluation of training programs and employee job combining professional skill appraisal [1].

\subsection{Lay Particular Stress on theory Examination and Operation Examination is Difficult to Practice}

Skill training can't work in the field operation, and has no suitable test platform. So the inspection can only be limited in the aspect of knowledge, and application assessment is difficult to practice.

Informatization and intellectualization will plays more and more important role in enterprise training evaluation with the development of information technology and virtual reality technology. [2] Information reflected in the computer multimedia technology and network technology as the core of technical level, and reflected in the staff quality assessment concept of modernization, namely the concept of system level. Intelligent development helps the enterprise training evaluation in relation to the work site more closely, more comprehensive evaluation of employees.

\section{The Oil Workers Professional Quality Inspection System Design}

\subsection{Design principles}

3.1.1. Practicality. It can be closely combining the actual requirement of oil gathering and transferring enterprise oil relief work, and convenient and flexible to implement training evaluation applications. It should have 
a test, examination, assessment, feedback, query and other functions.

3.1.2. Strong adaptability. According to the actual needs of oil workers training work, make the system have strong expansion, perfect function, meet different training programs and different levels of skill appraisal requirements.

3.1.3. fault-tolerant. System must have strong ability of fault tolerance. It should have the corresponding prompt function and processing power when data is error. Each processing link should have high reliability, safety and confidentiality.

3.1.4. Easy maintenance. Administrator permissions users can conveniently maintain system.

\subsection{Function Design}

Professional quality assessment system for Oil workers in oil gathering and transferring enterprise is in the service of professional skill appraisal and regular training examination, realize theoretical knowledge and skills appraisal, meet the independent test and the assessment of company(yard) unified requirements. In particular, including:

3.2.1 Theory knowledge examination system is based on dynamic database.

Employees can do knowledge self-test, enterprise can pass the examination for professional skill appraisal and training effect evaluation.

3.2.2 Standard operating procedures and process switching is based on virtual simulation evaluation system. It can test the standardization of operation and proficiency evaluation to staff.

\subsection{Function Module Design}

\subsubsection{Theory Test System Design}

Theory test system function includes questions bank establishment, set volume, examination and evaluation, feedback, check, etc. 1). The question bank system. Build the professional skill appraisal question bank system according to topic and professional skill level. 2). Self-test on theory knowledge. The employee can enter the system at any time to test by self, without having to transfer station/unified organization. 3). Examination to the training. The examination is carried out to evaluate training effect. Evaluation system can build paper automatically according to sets up the paper structure (including topic, score, subject number, level, etc.) by the lecturer, and can organize many people at the same time to exam. 4). Professional skills appraisal. It sets five levels of examination question bank according to the request of oil workers professional skill appraisal, including the primary works, intermediate class, senior worker, technician, senior technician. When employees do professional skills appraisal, evaluation system can build paper automatically according to the level required to extract the corresponding subject group volume test. 5). The paper reviews. Objective questions of test paper (multiple choices, true or false, fills up the topic) are scored by the system automatically, subjective topic are scored by the instructor. 6). The examination results feedback. Test paper provides the test feedback to employees after review, including grades, calibration answers and Suggestions, etc. Specific function structure as shown in Figure 1:

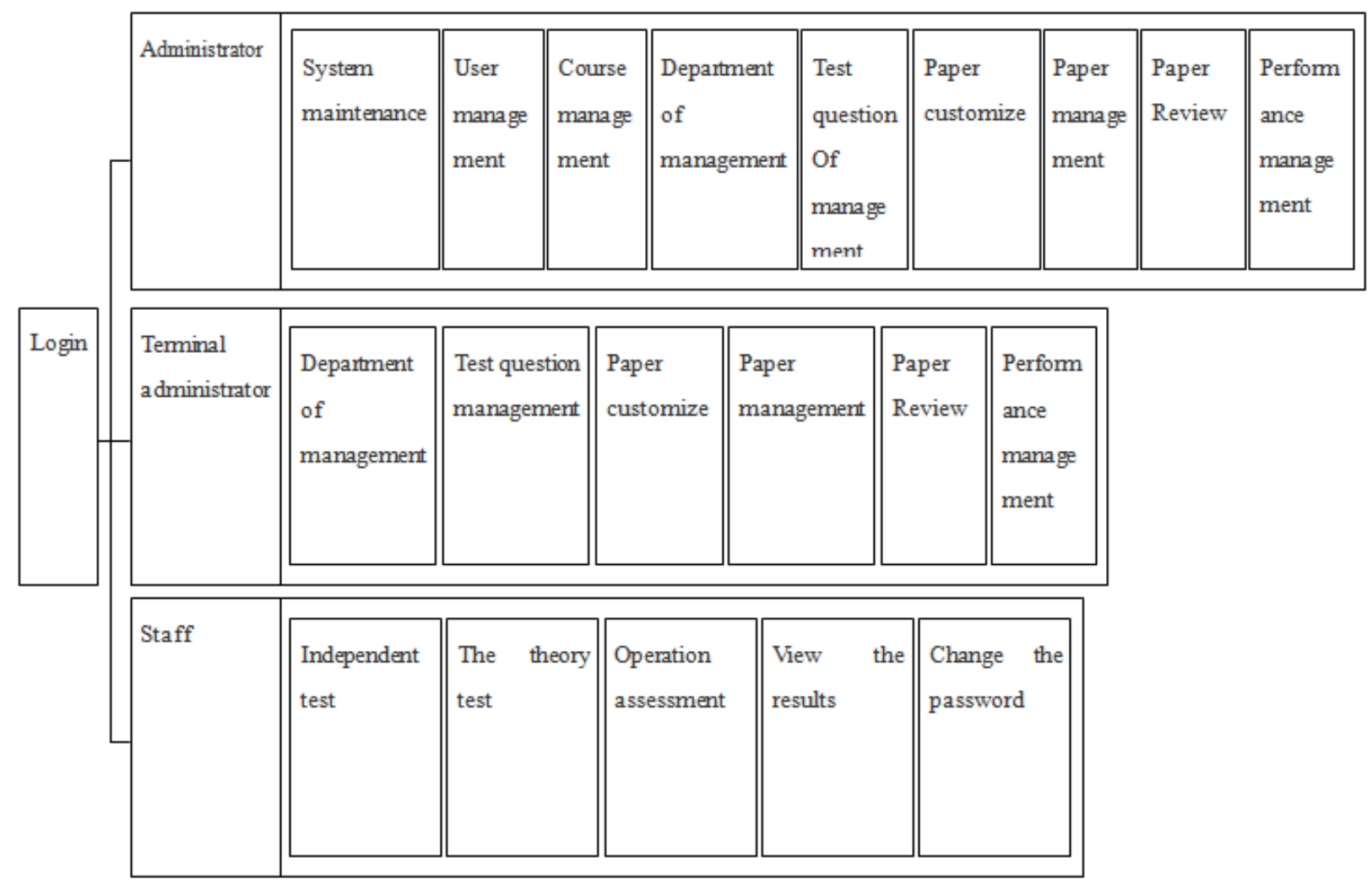

Figure 1. The function structure of the assessment system

\subsubsection{Operating Assessment System Design}

Operating assessment system simulate Oil road and works scene according to standard operating procedure 
card operation and standard operating procedures. System provides some functions, such as virtual interactions in the form of personification, key steps in action, theory and matters needing attention with text options to choose from, mistake operation to give feedback in time [3].

\section{Oil Workers Professional Quality Appraisal System Development}

\subsection{Technical Solution}

Theory test system adopts B/S mode as the overall architecture, using .NET and SQLSERVER2005. Rolebased process control, the system sets three different permissions, including employee, operations manager, the super administrator. According to the level of professional skills, the system accordingly designs five levels (primary works, intermediate class, senior worker, technician, senior technician) examination question bank, and use the algorithm of specific to select test questions to form paper.

Operating assessment system uses virtual reality technology to simulate Oil road and works scene. Equipment operates on the basis of personification of virtual interactions, and provides real-time feedback. The key technology of implementation includes: Scene modeling technology, scene, key equipment, oil and other entities were worked for $3 \mathrm{~d}$ modeling through the real measurement and the methods of shot; Virtual interactive technology, the application of virtual reality platform and programming language embedded in the ROUTE events controls interactive dynamic in the scene [4].

\subsection{The Implementation of Evaluation System}

\subsubsection{Theory Test System Implementation}

The system sets up three kinds of different operating permissions, including: Super administrator, it has the highest authority, and can manage all terminals to the administrator, and data operation permissions; Terminal administrator: belonging to a certain terminal, and it can only have operation permissions to this site data; Staff: belonging to a particular station, and it can only take part in the organization of examination. Next, as an administrator and ordinary staff identity respectively introduce the realization of the function of the system [5].

1. An administrator login system

Results show below Figure 2 when administrator login system.

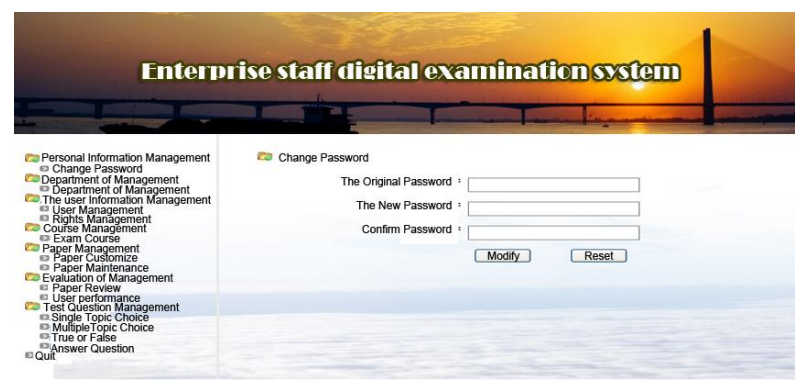

Figure 2. The working interface when administrator login system

System management: including department management, user management, subjects and the logged in user's password management and so on.
Questions bank management: management oil workers professional skill appraisal question bank management from topic and level, which can realize the increase, view, modification, and delete subject. The system supports four types of questions, including single choice, multiple choice questions, true or false question and essay question.

The examination paper management: according to the group strategy of generating test paper automatically and the management of the generated test paper. Administrators can custom paper structure, including the topic, score, number, levels, and the system forms paper according to the policies set by the administrators from random question selection in the question bank group, as shown in Figure 3. At the same time, it can provide view, delete, edit the status of display (True: employees can choose this paper exam, false: staff can't see this paper) the generated test paper.

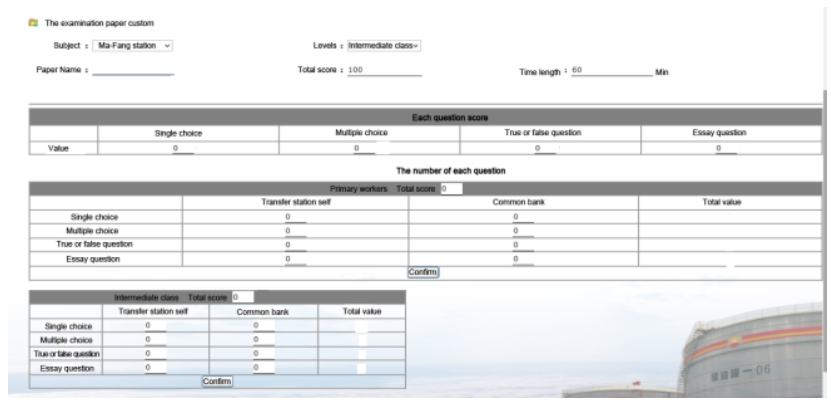

Figure 3. The working interface of the paper structure customized

Performance management: Test scores and student performance management. The system realizes Objective questions of test paper (single choice, multiple choices, true or false) are scored by the system automatically, subjective topic are scored by the instructor. The administrator can view the test scores.

2. Staff member login system

Results show below Figure 4 when staff member login system.

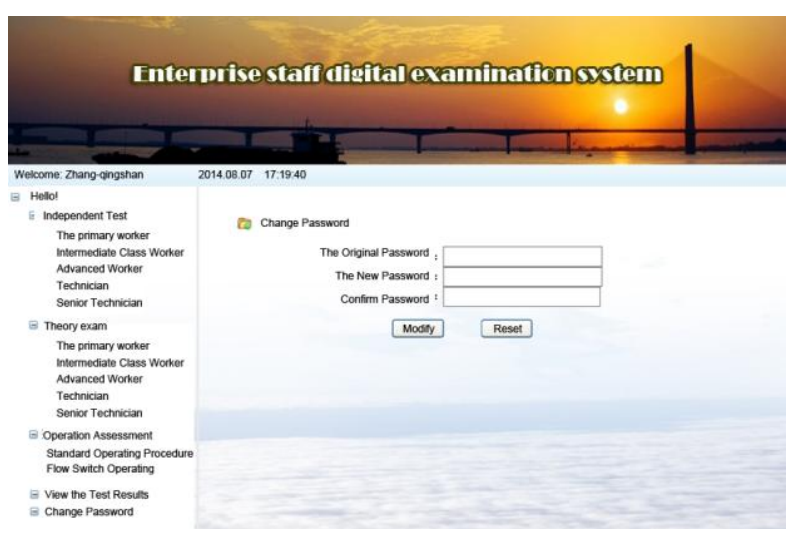

Figure 4. The working interface when staff member login system

Employees can choose different levels (primary works, intermediate class, senior worker, technician and higher technician) to test. The system provides feedback immediately test results after submitting the test.

Theory exam: the employee can choose different levels (primary works, intermediate class, senior worker, technician and higher technician) by opened. System has the function of the countdown. If the time of the exam had come to the end, the answers will automatically terminate and be submitted. 
Operation assessment: provide interface for operation assessment.

View test results: view scores and result feedback. The system provides the reference answer, and red marked wrong topic.

\subsubsection{Assessment of Operating System}

1. Virtual simulation operation assessment for standard operating procedure

The system uses modular design according to the standard operating procedures and script, check at the piece of sequential operations, and a strict time limit. [6] If any one of the key steps is error in operation that the entire project result is zero, and immediately terminate the examination. Standard for centrifugal pump start-up operation assessment project below Figure 5, that is divided into six modules. When every operating is completed that system can be immediate scoring reality in the left upper corner of the screen. It need click on the "submit" button when finish the whole project operation, and show the total score of the assessment.

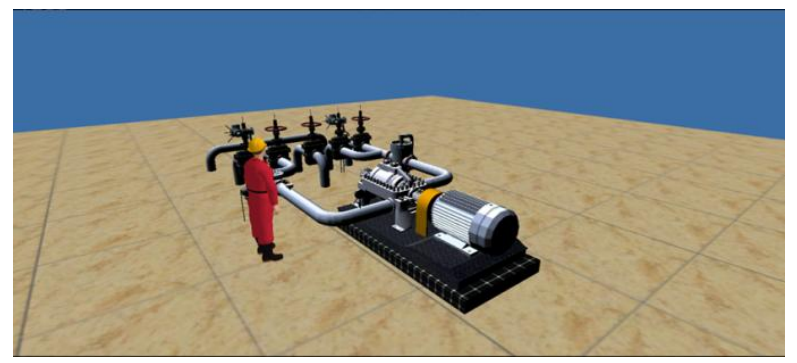

Figure 5. Centrifugal pump start-up operation assessment project

2. Flow switch operating virtual simulation operation assessment project

Assessment checks at the piece of sequential operations, and a strict time limit. If any one of the key steps is error in operation that the entire project result is zero, and immediately terminate the examination. System has the function of the countdown. If the time of the exam had come to the end, the answers will automatically terminate and be submitted. Figure 6 for oil into the pot to process switching operation assessment project.

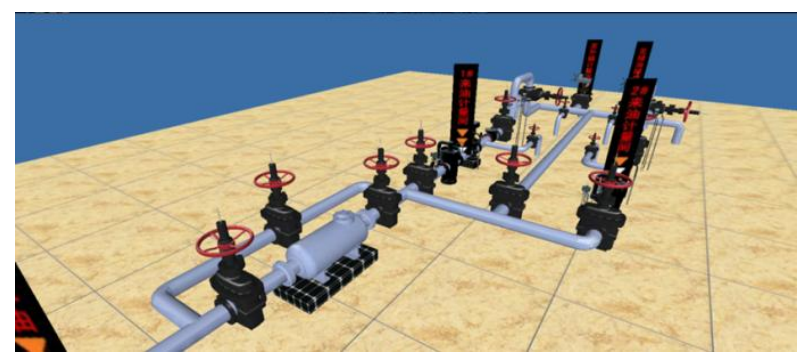

Figure 6. Oil into the pot to process switching operation assessment project

\section{System Application}

Chang-Qing oilfield company and Jiang-Han oilfield company imported the system, finished the question bank construction, and organized the new staff training and the professional skill appraisal. Details see Table 1. Chang-
Qing oilfield company build question bank with 5620 exam question, organization of 510 new employees training appraisal and 324 professional skill appraisal. Jiang-Han oilfield company build question bank with 4300 exam question, organization of 464 new employees training appraisal and 284 professional skill appraisal. Practice shows that the system fully functional, stable running, safe and reliable, and basically meets the requirements of enterprise staff appraisal.

Table 1. System application

\begin{tabular}{|c|c|c|c|}
\hline & $\begin{array}{c}\text { The question } \\
\text { bank } \\
\text { (quantity) }\end{array}$ & $\begin{array}{c}\text { New employees } \\
\text { training } \\
\text { appraisal(M) }\end{array}$ & $\begin{array}{c}\text { Professional } \\
\text { skill } \\
\text { appraisal(M) }\end{array}$ \\
\hline $\begin{array}{c}\text { Chang-Qing } \\
\text { oilfield } \\
\text { company }\end{array}$ & 5620 & 510 & 324 \\
\hline $\begin{array}{c}\text { Jiang-Han } \\
\text { oilfield } \\
\text { company }\end{array}$ & 4300 & 464 & 284 \\
\hline
\end{tabular}

\section{Conclusion}

The main content of this paper is design and development for oil gathering and transferring oil workers professional quality appraisal system. System is in the service of oil workers professional quality requirement and regular training appraisal, realize the two aspects of theoretical knowledge and operation skills examination, and satisfy the unified examination of enterprise organization, and satisfy the employee's independent test. Research and development of evaluation system is of great significance for organization and employee development.

Make staff appraisal organization, management more convenient and efficient.

Reduce the cost of employees' professional quality inspection.

Solve the employees' work - take an examination of "disconnect and time on the conflict.

Let the employee's training and combine examination to improve the training effect.

Build a comprehensive assessment system about the theoretical knowledge test and operation skills examination.

\section{References}

[1] Ren Wei-Jian, Application of Virtual Reality Technology in Simulation Training of Transportation and Storage of Oil and Gas. Journal of system simulation, 2005(6), 33-34.

[2] M Garland, P S Heckbert. Surface simplification using quadric error metrics.PhD thesis, CS Department, Carnegie Mellon University, 1998

[3] Wang Zhi-gang, Research on Training System Oriented Modern Manufacturing Enterprises. Journal of Xingtai Polytechnic College, 2011(7), 58-60.

[4] Lin Lin. Design of Training System of Oil Field Simulation Based on Virtual Reality Technology. Computer technology and development, 2012(10), 205-208.

[5] Tu Xiao-yuan. Artificial Animals for Computer Animation: Biomechanics, Locomotion, Perception and Behavior. Toronto: University of Toronto, 1996.

[6] Hao Hai-liang. Design of simulation training system for oil extraction. Mechanical \& electrical engineering magazine, 2008(1), 18-21. 